\title{
Estudo termoquímico assistido por computador para a produção de biodiesel
}

\section{Estudio termoquímico asistido por computadora para la producción de biodiésel}

\section{Thermochemical computer-aided study for biodiesel Production}

\author{
Jeffrey Leon-Pulido ${ }^{1 *}$; William Steve Fajardo-Moreno1; Mary Judith Arias-Tapia2; Angel Dario Gonzalez- \\ Delgado3; Viktor Oswaldo Cárdenas-Concha4; José Roberto Nunhez ${ }^{5}$ \\ 'Departamento de Procesos, Universidad EAN, Bogotá-Colombia* \\ ${ }^{2}$ Programa de Ingeniería Química, Universidad Tecnológica de Bolívar, Cartagena-Colombia \\ ${ }^{3}$ Departamento de Ingeniería Química, Universidad de Cartagena, Cartagena-Colombia \\ ${ }^{4}$ Laboratório de Biotecnologia e Sistemas Naturais, Universidade Federal de São Paulo, SP-Brasil \\ ${ }^{5}$ Faculdade de Engenharia Química, Universidade Estadual de Campinas, SP-Brasil \\ *jleonp@universidadean.edu.co
}

\begin{abstract}
Resumo
A importância de melhorar os processos de transformação industrial para processos mais eficientes é parte dos desafios atuais. Específicamente, o desenvolvimento de processos mais eficientes na produção de biocombustíveis, onde os processos de reação e separação podem ser intensificados, é de grande interesse para reduzir o consumo de energia associado ao processo. No caso do Biodiesel, o processo é definido por uma reação química e pelos componentes associados ao processo, onde o estudo termoquímico busca desenvolver cálculos para o entendimento subsequente do processo de reação e purificação. Assim, a análise da mistura dos componentes usando o simulador de processo Aspen Plus V9® descreve o estudo termoquímico. O método termodinâmico UNIFAC-DMD foi utilizado para estimar os parâmetros binários de equilíbrio dos reagentes usando o simulador. Os aspectos analisados apresentam o comportamento dos componentes em diferentes condições de temperatura, o comportamento azeotrópico e as condições termoquímicas determinadas.
\end{abstract}

Palavras-chave: UNIFAC-DMD; Termoquímica; Equilíbrio; Simulação; Biodiesel.

\section{Resumen}

La importancia de mejorar los procesos de transformación industrial a otros más eficientes hace parte de los desafíos de estos tiempos. Específicamente, el desarrollo de procesos mas eficientes en la producción de biocombustibles, en donde puedan ser intensificados los procesos de reacción y separación, son de gran interés para disminuir el consumo de energía asociado al proceso. En el caso del Biodiésel, el proceso es definido por una reacción química y los componentes asociados al proceso, donde el estudio termoquímico busca desarrollar calculos para el posterior entendimiento del proyecto de reacción y purificación. Siendo así, el análisis de los componentes mezclados haciendo uso del simulador de procesos Aspen Plus $V 9 \circledast$ describe el estudio termoquímico. Para ello, el método termodinámico UNIFAC-DMD fué utilizado para estimar los parámetros binarios del equilibrio de los reactivos y reactantes calculados con el simulador. La observación de los aspectos analizados presenta el comportamiento de los componentes a diferentes condiciones de temperatura, el comportamiento azeotrópico y condiciones termoquímica fueron determinados.

Palabras clave: UNIFAC-DMD; Termoquímica; Equilibrio; Simulación; Biodiésel.

Cita: Leon-Pulido J, Fajardo Moreno WS, Tapias Arias ML, Gonzalez-Delgado AD, Cárdenas Concha VO, Nunhez JR. Estudo Termoquímico Assistido por Computador para a Produção de Biodiesel. rev.ion. 2019;32(2):77-84. doi:10.18273/revion.v32n2-2019008 
rev.ion. 2019;32(2):77-84. Bucaramanga (Colombia).

\begin{abstract}
The importance of improving industrial transformation processes to more efficient processes ones is part of the challenges of these times. Specifically, the development of more efficient processes in the production of biofuels where the reaction and separation processes can be intensified are of great interest to reduce the energy consumption associated with the process. In the case of Biodiesel, the process is defined by a chemical reaction and the components associated with the process, where the thermochemical study seeks to develop calculations for the subsequent understanding of the reaction and purification project. Thus, the analysis of the mixed components using the Aspen Plus V9® process simulator describes the thermochemical study. For this, the UNIFAC-DMD thermodynamic method was used to estimate the binary parameters of the equilibrium of the raw materials and reactants calculated with the simulator. The observation of the analyzed aspects shows the behavior of the components at different temperature conditions, the azeotropic behavior and thermochemical conditions were determined.
\end{abstract}

Keywords: UNIFAC-DMD; Thermochemical; Equilibrium; Simulation; Biodiesel.

\section{Introdução}

Diversos estudos têm sido desenvolvidos ao longo do mundo para aprimorar o conhecimento dos biocombustíveis [1-3], sedo a maior parte das pesquisas dirigidas ao estudo da combustão química, a cinética e o comportamento do processo químico [4]. De forma geral, a produção de biodiesel é definida pela reação química dos componentes, pelas tecnologias e pelos catalisadores que potencializam o processo [5].

A intensificação de processos (Process Intensification - PI) faz parte do desenvolvimento dos processos de transformação e manufatura, o conceito contribui na diminuição do tamanho dos processos industriais, visando a integração de processos, unidades modulares e compactas [6]. No presente trabalho, um estudo termoquímico dos componentes puros e em mistura são desenvolvidos para compreender a fenomenologia da produção do biodiesel.

\section{O Biodiesel}

O biodiesel é definido como um combustível líquido sintético de fonte renovável, sua constituição básica é uma mistura de ésteres alquílios de ácidos graxos de cadeia longa obtido de óleos vegetais segundo a American Society for Testing Materials - ASTM.

De forma geral, o biodiesel é obtido a partir da reação de transesterificação, esta consiste na reação de álcool e triglicerol para a formação de ésteres e glicerina [7]. A reação pode ser acelerada por catalisadores ácidos, básicos, enzimáticos ou especiais [8]. O processo de transesterificação é considerado como o método mais eficaz para a transformação de óleos vegetais, por apresentar praticidade e melhoras das propriedades para o uso como combustível [9].

As reações químicas precisam de dados cinéticos, a cinética do biodiesel é chamada como elementar quando a ordem da reação coincide com a molecularidade. $O$ estudo cinético da reação de transesterificação, bem sendo estudado por diferentes centros de pesquisa, assim como, o comportamento da reação, a oxidação, a velocidade de reação e condições de operação como a temperatura $[10,11]$.

O biodiesel é obtido geralmente a partir de óleos vegetais, de modo que possui caraterísticas biodegradáveis para processos mais rigorosos sim toxicidade. Durante o processo de produção convencional, o óleo é misturado com hidróxidos de sódio ou potássio e um álcool. Os produtos da reação obtidos são ésteres metílicos ou etílicos (biodiesel) e glicerol como subproduto [12].

\section{Caraterísticas do Biodiesel}

$\mathrm{Na}$ atualidade, as necessidades de alternativas de combustíveis substitutos fazem do biodiesel uma grande possibilidade, no entanto, a produção de matéria-prima de óleos vegetais não é suficiente, contudo, existem inúmeras justificativas para o desenvolvimento de processos de biodiesel, entre elas: a substituição do diesel por biodiesel pode ser realizada na maioria dos equipamentos movidos a diesel, diminui a emissão de gases, o biodiesel é não-tóxico, biodegradável, entre outras [13]. Esta necessidade apresenta um desafio no balanço entre agricultura, meio ambiente y acréscimo económico.

As propriedades e caraterísticas do biodiesel são apresentadas na Tabela 1. 
O biodiesel pode ser obtido por diferentes geral para a produção é apresentado na Figura 1 e caminhos, transesterificação ou esterificação a caracterizado, na sequencia, no Quadro 1:

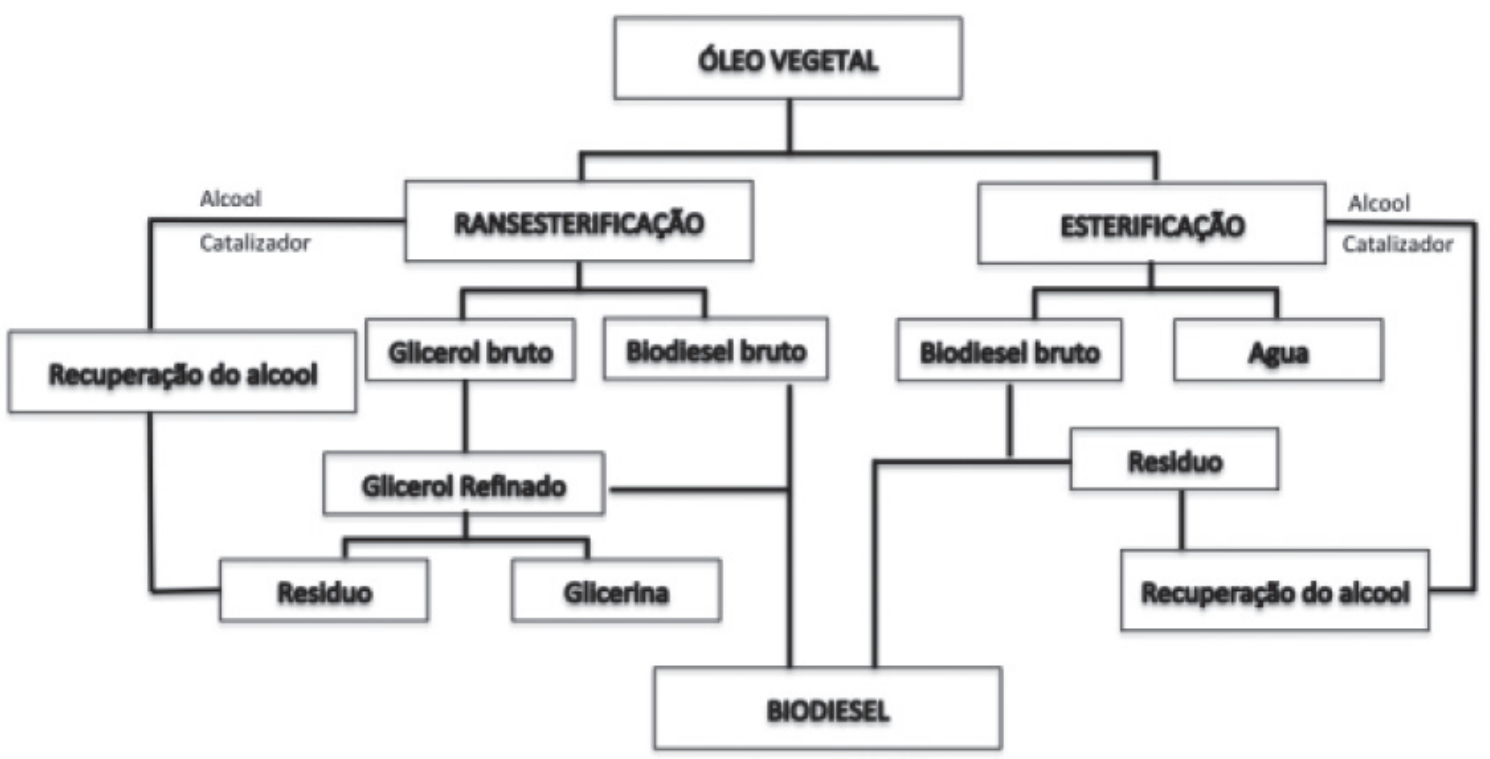

Figura 1. Diagrama geral de produção de biodiesel a partir de óleo vegetal.

Tabela 1. Caraterísticas do biodiesel.

\begin{tabular}{|c|c|}
\hline Nome químico & Éster (m)etílico de ácido graxo \\
\hline Intervalo da fórmula química & $\mathrm{C}_{14}-\mathrm{C}_{24}(\mathrm{~m})$ etil éster ou $\mathrm{C}_{15-25} \mathrm{H}_{28-48} \mathrm{O}_{2}$ \\
\hline $\begin{array}{l}\text { Intervalo de viscosidade cinemática }\left(\mathrm{mm}^{2} / \mathrm{s} \text { a }\right. \\
\qquad 313 \mathrm{~K})\end{array}$ & $3,3-5,2$ \\
\hline Intervalo de densidade (kg/m³ a $288 \mathrm{~K})$ & $860-894$ \\
\hline Intervalo do ponto de ebulição (K) & $>475$ \\
\hline Intervalo do ponto de fulgor (K) & $420-450$ \\
\hline Intervalo de destilação (K) & $470-600$ \\
\hline Pressão de vapor (mmHg a $295 \mathrm{~K}$ ) & $<5$ \\
\hline $\begin{array}{c}\text { Solubilidade em água/Aparência física/Odor/ } \\
\text { Biodegradabilidade/Reatividade }\end{array}$ & $\begin{array}{l}\text { Insolúvel em água/De amarelo claro a escuro, líquido } \\
\text { límpido/Odor levemente azedo/semelhante a sabão/ } \\
\text { Mais biodegradável que o diesel de petróleo/Estável, mas } \\
\text { deve-se evitar o contato com agentes oxidantes fortes. }\end{array}$ \\
\hline
\end{tabular}

Fonte: Adaptado de Demirbas (2009) 
rev.ion. 2019;32(2):77-84. Bucaramanga (Colombia).

Quadro 1. Tecnologias para a produção de Biodiesel.

\begin{tabular}{|c|c|c|c|c|}
\hline Variável & Catálise Alcalina & $\begin{array}{c}\text { Catálise Enzimática } \\
\text { (Lípase) }\end{array}$ & Álcool Supercrítico & Catálise Ácida \\
\hline $\begin{array}{l}\text { Temperatura da reação } \\
\text { (K) }\end{array}$ & $333-343$ & $303-313$ & $512-658$ & $328-353$ \\
\hline $\begin{array}{c}\text { Ácidos graxos livres } \\
\text { na MP }\end{array}$ & Produtos Saponificados & Ésteres metílicos & Ésteres & Ésteres \\
\hline Água na MP & Interfere na reação & Não tem influência & - & $\begin{array}{l}\text { Interfere na } \\
\text { reação }\end{array}$ \\
\hline Rendimento Ésteres & Normal & Alta & Boa & Normal \\
\hline $\begin{array}{c}\text { Recuperação de } \\
\text { Glicerol }\end{array}$ & Difícil & Fácil & - & Difícil \\
\hline $\begin{array}{l}\text { Purificação dos } \\
\text { Ésteres Metílicos }\end{array}$ & Lavagem & Nenhuma & - & Lavagem \\
\hline
\end{tabular}

Fonte: Adaptado de SHARMA et al. (2008). *MP -Matéria Prima

\section{Método UNIFAC-DMD}

Assim, uma modificação do modelo base é o UNIFAC-DMD, este modelo inclui uma melhor predição do calor da mistura, relação de dependência com a temperatura e parâmetros simples para o equilbro líquido-vapor e líquidolíquido (ELV - ELL). Para o desenvolvimento dos cálculos, basta ter informação das propriedades termodinâmicas como: tipo de mistura, coeficiente de fugacidade, energia de Gibbs, entalpia, entropia e densidade. Na modificação realizada para o modelo UNIFAC-DMD, o desenvolvimento do cálculo é significativamente diferente na combinação das partes, A dependência da temperatura é descrita para uma equação de interação dos parâmetros [14].

Basicamente o modelo apresenta uma modificação combinatória quando comparado com o UNIFAC original [15-17] onde a dependência da temperatura na interação dos parâmetros é:

$$
\ln \gamma_{\mathrm{i}}^{\mathrm{C}}=\ln \left(\frac{\Phi_{i}^{\prime}}{\mathrm{x}_{\mathrm{i}}}\right)+1-\frac{\Phi_{\mathrm{i}}}{\mathrm{x}_{\mathrm{i}}}-\frac{z}{2} q_{i}\left(\ln \frac{\Phi_{\mathrm{i}}}{\theta_{i}}+1-\frac{\Phi_{\mathrm{i}}}{\theta_{i}}\right)
$$

Quando:

$$
\frac{\Phi_{i}^{\prime}}{x_{i}}=\frac{r_{i}^{3 / 4}}{\Sigma_{j} x_{j} r_{j}^{3 / 4}}
$$

A dependência é dada pela temperatura como:

$$
\tau_{\mathrm{mn}}=\mathrm{e}^{-\mathrm{a}_{\mathrm{mn}} / \mathrm{T}}
$$

e,

$$
a_{m n}=a_{m n, 1}+a_{m n, 2} T+a_{m n, 3} T^{2}
$$

Os parâmetros usados no simulador são: UFGRPD $\left(k, \quad m, \quad v_{m}, v_{k}\right) ; \quad \operatorname{GMUFDQ}\left(\mathrm{Q}_{k}\right) ; \quad \operatorname{GMUFDR}\left(\mathrm{R}_{k}\right)$; UNIFDM/1 $\left(\mathrm{a}_{\text {min } 1}\right) ; U N I F D W / 2\left(\mathrm{a}_{\text {min } 2}\right) ; \operatorname{UNIFDM} / 3\left(\mathrm{a}_{\min 3}\right)$.

\section{Considerações gerais}

O sistema de estudo envolve os seguintes compostos: Metanol $\left(\mathrm{CH}_{4} \mathrm{O}\right)$, Trioleína $\left(\mathrm{C}_{57} \mathrm{H}_{104} \mathrm{O}_{6}\right)$, Trimiristina $\left(\mathrm{C}_{45} \mathrm{H}_{86} \mathrm{O}_{6}\right)$, Metil oleato $\left(\mathrm{C}_{19} \mathrm{H}_{36} \mathrm{O}_{2}\right)$, glicerol $\left(\mathrm{C}_{3} \mathrm{H}_{8} \mathrm{O}_{3}\right), \quad \mathrm{NaOH}$, agua, Tripalmitico $\left(\mathrm{C}_{51} \mathrm{H}_{98} \mathrm{O}_{6}\right)$ e Metil Palmitato $\left(\mathrm{C}_{17} \mathrm{H}_{34} \mathrm{O}_{2}-\mathrm{N} 1\right)$ como principais componentes para o biodiesel. modelos termodinâmicos são de grande importância nos cálculos de propriedades termodinâmicas, dentro dos principais modelos termodinâmicos encontrados na base do Aspen Plus. Sabe-se que a representação de um sistema é fundamentada por um modelo termodinâmico e dos dados de entrada, por tanto, a data dos componentes puros e propriedades termodinâmicas são absolutamente necessários para simular o processo. O simulador de processos Aspen Plus $\mathrm{V} 9 \AA$ contém as informações necessárias para a simulação do sistema (biodiesel).

A análise termodinâmica determina o déficit da entalpia líquida e as perdas exegéticas devido à irreversibilidade (resultado da entropia) em cada estágio da coluna. Assim, o estudo da unidade de processo desde a ótica dos pontos, onde não se apresenta distribuição uniforme, merece melhorias. A redução da irreversibilidade permite o aumento do trabalho potencial disponível na unidade de processo, em proporção, conseguese uma diminuição do consumo energético e um aumento da eficiência, suportada no conceito de um estado termodinâmico mínimo (ETM).

A metodologia adotada para o design do processo se fundamenta na obtenção do biodiesel em uma unidade intensificada de reação e separação. Assim, o estudo termodinâmico permite o entendimento dos fenómenos onde acontece a síntese do biodiesel.

\section{Propriedades da Síntese do Biodiesel}

As propriedades dos componentes envolvidos 
na produção do biodiesel foram estimadas no simulador de processos e são apresentados nas Tabela 2a, 2b.

As condições padrão, fora definidas com base a experimentos reportados pelos autores em condições ideais e reais na base de dados do simulador. Assim, propriedades como o massa molecular e condições críticas são levadas em consideração para atingir o estudo termoquímico. A reação de transesterificação acontece na presença de álcool e catalisador $\mathrm{NaOH}$, o excesso do álcool é considerado para garantir a produção do biodiesel. Em consideração ao desenvolvimento de projeto conceitual da unidade intensificada de produção com aproveitamento de energia, a separação dos ácidos graxos (Fatty acid methyl ester - FAME) não será desenvolvida, entanto, a obtenção dos FAME serão o objetivo principal a ser atingido no trabalho.

A pressão de vapor dos componentes envolvidos no processo do biodiesel descreve o comportamento da pressão da fase vapor sobre a fase líquida. É estudado tambem, o equlíbro dinâmico que acontece em uma determinada temperatura, no caso do biodiesel, a pressão de vapor dos catalisadores (Equação de Antoine. Os parâmetros para a obtenção destas variávies, para cada componente são apresentados na Tabela 3.

Tabela 2a. Propriedades dos Componentes - Síntese BIODIESEL.

\begin{tabular}{|c|c|c|c|c|c|c|c|c|c|}
\hline $\begin{array}{l}\text { Propriedade/ } \\
\text { Unidade }\end{array}$ & METHANOL & O०० & METHYL-O & GLYCEROL & NAOH & WATER & H3PO4 & NA3PO4 & METHYL-P \\
\hline $\begin{array}{l}\text { Gibbs Padrão de } \\
\text { Formação GI (J/ } \\
\text { kmol) }\end{array}$ & $-1,63 E+08$ & $-2,97 \mathrm{E}+8$ & $-1,17 E+08$ & $-4,47 E+08$ & $-2,29 E+08$ & $-2,29 E+08$ & $-2,29 E+08$ & $-2,29 E+08$ & $-2,54 E+08$ \\
\hline $\begin{array}{l}\text { Entalpia Padrão de } \\
\text { Formação GI (J/ } \\
\text { kmol) }\end{array}$ & $-2,01 E+08$ & $-2,02 E+9$ & $-6,26 E+08$ & $-5,78 E+08$ & $-2,42 E+08$ & $-2,42 E+08$ & $-2,42 E+08$ & $-2,42 E+08$ & $-7,01 E+08$ \\
\hline $\begin{array}{c}\text { Entalpia de } \\
\text { Vaporização (J/ } \\
\text { kmol) }\end{array}$ & $3,53 \mathrm{E}+07$ & - & $6,39 \mathrm{E}+07$ & $6,64 \mathrm{E}+07$ & $4,07 \mathrm{E}+07$ & 4,07E+07 & 4,07E+07 & $4,07 \mathrm{E}+07$ & $5,75 \mathrm{E}+07$ \\
\hline $\begin{array}{l}\text { Entalpia padrão de } \\
\text { combustão (J/kmol) }\end{array}$ & $-6,38 E+08$ & $-3,28 E+10$ & $-1,11 \mathrm{E}+10$ & $-1,48 E+09$ & 0 & 0 & 0 & 0 & $-9,96 E+09$ \\
\hline massa Molecular & 32,0422 & 885,449 & 296,494 & 92,0947 & - & 18,0153 & - & - & 270,456 \\
\hline $\begin{array}{l}\text { Pressão Critica (N/ } \\
\left.\qquad m^{\wedge} 2\right)\end{array}$ & $8,08 \mathrm{E}+06$ & 322432 & $1,28 \mathrm{E}+06$ & $7,50 \mathrm{E}+06$ & $2,21 \mathrm{E}+07$ & $2,21 \mathrm{E}+07$ & $2,21 \mathrm{E}+07$ & $2,21 \mathrm{E}+07$ & $1,24 \mathrm{E}+06$ \\
\hline $\begin{array}{c}\text { Gravidade } \\
\text { Específica Padrão }\end{array}$ & 0,7962 & 0,911369 & 0,872404 & 1,26124 & 1 & 1 & 1 & 1 & 0,869994 \\
\hline $\begin{array}{l}\text { Temperatura de } \\
\text { Ebulição (K) }\end{array}$ & 337,85 & 813,739 & 617 & 561 & 373,15 & 373,15 & 373,15 & 373,15 & 597,7 \\
\hline $\begin{array}{l}\text { Temperatura Crítica } \\
\text { (K) }\end{array}$ & 512,5 & 943,23 & 764 & 850 & 647,096 & 647,096 & 647,096 & 647,096 & 00762,2 \\
\hline VB (cum/kmol) & 0,0427452 & 2,70798 & 0,489087 & 0,08685 & 0,0188311 & 0,0188311 & 0,0188311 & 0,0188311 & 0,442809 \\
\hline $\begin{array}{l}\text { Volume Crítico } \\
\text { (cum/kmol) }\end{array}$ & 0,117 & 3,25102 & 1,06 & 0,264 & 0,0559472 & 0,0559472 & 0,0559472 & 0,0559472 & 1,15 \\
\hline $\begin{array}{c}\text { Fator de } \\
\text { Compressibilidade } \\
\text { Crítica }\end{array}$ & 0,222 & 0,107 & 0,214 & 0,28 & 0,229 & 0,229 & 0,229 & 0,229 & 0,224 \\
\hline
\end{tabular}


rev.ion. 2019;32(2):77-84. Bucaramanga (Colombia).

Tabela 2b. Propriedades dos Componentes - Síntese BIODIESEL.

\begin{tabular}{|c|c|c|c|c|c|c|c|c|c|}
\hline Propriedade/Unidade & METHYL-M & METHYL-S & $1-0$ & МMM & PPP & PPS & PPO & POS & MMP \\
\hline $\begin{array}{l}\text { Entalpia Padrão de } \\
\text { Formação GI (J/kmol) }\end{array}$ & $-6,44 E+08$ & $-7,42 E+08$ & $-1,16 E+09$ & $-2,07 E+09$ & $-2,14 \mathrm{E}+09$ & $-2,18 E+09$ & $-2,10 E+09$ & $-2,14 \mathrm{E}+09$ & $-2,09 \mathrm{E}+9$ \\
\hline $\begin{array}{c}\text { Entalpia de } \\
\text { Vaporização (J/kmol) }\end{array}$ & $5,44 \mathrm{E}+07$ & $6,06 \mathrm{E}+07$ & $7,09 \mathrm{E}+07$ & - & - & - & - & - & - \\
\hline $\begin{array}{l}\text { Entalpia padrão de } \\
\text { combustão (J/kmol) }\end{array}$ & $-8,81 E+09$ & $-1,12 \mathrm{E}+10$ & $-1,19 E+10$ & - & - & - & - & - & - \\
\hline massa Molecular & 242,402 & 298,51 & 356,546 & 723,174 & 807,336 & 835,389 & 833,373 & 861,427 & 751,228 \\
\hline Pressão Crítica (N/m^2) & $1,42 \mathrm{E}+06$ & $1,08 \mathrm{E}+06$ & $1,01 \mathrm{E}+06$ & 418300 & 366874 & 352837 & 350433 & 337854 & 399391 \\
\hline $\begin{array}{c}\text { Gravidade Específica } \\
\text { Padrão }\end{array}$ & 0,865421 & 0,868064 & 0,953565 & 0,904398 & 0,906716 & - & - & - & - \\
\hline $\begin{array}{l}\text { Temperatura de } \\
\text { Ebulição (K) }\end{array}$ & 569,9 & 623,7 & 647,017 & 784,847 & 794,264 & 800,847 & 800,847 & 807,339 & 788,187 \\
\hline Temperatura Crítica (K) & 741,2 & 781,1 & 815,794 & 898,558 & 923,368 & 930,937 & 930,234 & 937,526 & 907,211 \\
\hline $\begin{array}{l}\text { Volume Crítico (cum/ } \\
\text { kmol) }\end{array}$ & 0,993 & 1,31 & 1,25028 & 2,61337 & 2,94793 & 3,05945 & 3,04896 & 3,16048 & 2,72489 \\
\hline $\begin{array}{c}\text { Fator de } \\
\text { Compressibilidade } \\
\text { Crítica }\end{array}$ & 0,229 & 0,219 & 0,204 & 0,141128 & 0,197881 & & & & \\
\hline
\end{tabular}

Tabela 3. Parâmetros para estimação da pressão de vapor da Equação de Antoine.

\begin{tabular}{cccc}
\hline Componente & $\mathrm{NaOH}$ & $\mathrm{H}_{3} \mathrm{PO}_{4}$ & $\mathrm{Na}_{3} \mathrm{PO}_{4}$ \\
\hline Temperatura & $\mathrm{C}$ & & \\
$\mathbf{1}$ & $-1,00 \mathrm{E}+20$ & $-31,5129$ & $-1,00 \mathrm{E}+20$ \\
$\mathbf{2}$ & 0 & 0 & - \\
$\mathbf{3}$ & 273,15 & 273,15 & 273,15 \\
$\mathbf{4}$ & 0 & 0 & - \\
$\mathbf{5}$ & 0 & 0 & - \\
$\mathbf{6}$ & 0 & 0 & - \\
$\mathbf{7}$ & 0 & 0 & - \\
$\mathbf{8}$ & $-273,15$ & $-0,15$ & - \\
$\mathbf{9}$ & 1726,85 & 199,85 & - \\
\hline
\end{tabular}

Para caraterizar a equação de potência, foram levadas as considerações a seguir: a equação foi descrita em concentração molar, o fator de temperatura exponencial é considerado zero, as características para o fator pré-exponencial e energia de ativação foram obtidos do trabalho de Narvaez [18], quem apresentou uma proposta da cinética da transesterificação para o óleo de palma.

\section{Determinação dos Parâmetros do Equilíbrio} Os modelos termodinâmicos avaliados foram UNIFAC, UNIFAC-DMD UNIQUAC, NRTL, PENG ROBINSON. Uma vez estudadas as propriedades dos componentes e as fases presentes na operação foi escolhido o método UNIFAC-DMD para a síntese do Biodiesel. Este além de ser sugerido na literatura é comprovado pelo meio do método curto de análise "property method selection" do simulador Aspen Plus V9®. O UNIFAC-DMD usa a equação de estado Rendlish-Kwong-Soave, suportado no método RK-Soave, este é calculado pelo equilíbrio binário e determinado pelas fases com restrição de temperatura mínima de $290 \mathrm{~K}$ $\left(16^{\circ} \mathrm{C}\right)$ e máxima de $420 \mathrm{~K}\left(146.85^{\circ} \mathrm{C}\right)$, o método, inclui maior dependência entre os parâmetros e a temperatura e calor de mistura [19-21].

Os componentes envolvidos foram estimados através do simulador. Para facilitar os cálculos de estimação de parâmetros a ferramenta Estimate all missing parameters foi usada para os trabalhos do equilíbrio. Os cálculos das propriedades físicas e seu comportamento no equilíbrio foi suportado no modelo Rendlich Kwong-Soave e NRTL para o tratamento geral com o UNIFAC - DMD. Os parâmetros estimados são apresentados na Tabela 4. 
rev.ion. 2019;32(2):77-84. Bucaramanga (Colombia).

Tabela 4. Propriedades dos Componentes - Síntese TAME.

\begin{tabular}{cccccccccc}
\hline $\begin{array}{c}\text { Componente } \\
\mathbf{i}\end{array}$ & METHANOL & METHANOL & METHANOL & METHANOL & METHANOL & GLYCEROL & GLYCEROL & GLYCEROL & GLYCEROL \\
\hline $\begin{array}{c}\text { Componente } \\
\mathbf{j}\end{array}$ & GLYCEROL & NAOH & WATER & H3PO4 & NA3PO4 & NAOH & WATER & H3PO4 & NA3PO4 \\
\hline $\begin{array}{c}\text { Temperatura } \\
\text { AIJ }\end{array}$ & 0 & $-0,693$ & $-0,693$ & $-0,693$ & $-0,693$ & $-0,7318$ & $-0,7318$ & $-0,7318$ & $-0,7318$ \\
AJI & 0 & 2,7322 & 2,7322 & 2,7322 & 2,7322 & $-1,2515$ & $-1,2515$ & $-1,2515$ & $-1,2515$ \\
BIJ & 547,83 & 172,987 & 172,987 & 172,987 & 172,987 & 170,917 & 170,917 & 170,917 & 170,917 \\
BJI & $-218,522$ & $-617,269$ & $-617,269$ & $-617,269$ & $-617,269$ & 272,608 & 272,608 & 272,608 & 272,608 \\
CIJ & 0,3 & 0,3 & 0,3 & 0,3 & 0,3 & 0,3 & 0,3 & 0,3 \\
DIJ & 0 & 0 & 0 & 0 & 0 & 0 & 0 & 0 \\
EIJ & 0 & 0 & 0 & 0 & 0 & 0 & 0 & 0 \\
EJI & 0 & 0 & 0 & 0 & 0 & 0 & 0 & 0 \\
FIJ & 0 & 0 & 0 & 0 & 0 & 0 & 0 & 0 \\
FJI & 0 & 0 & 0 & 0 & 0 & 0 & 0 & 0 \\
TLOWER & 25 & 24,99 & 24,99 & 24,99 & 24,99 & 25 & 25 & 0 \\
TUPPER & 62,5 & 100 & 100 & 100 & 100 & 290 & 290 & 290 \\
\hline
\end{tabular}

Propriedades termo-físicas para os tri, di e mono glicerídeos foram obtidos da base de dados para o biodiesel, disponíveis no simulador. O estudo do sistema apresenta um azeotropo à temperatura de $726,45 \mathrm{~K}\left(453^{\circ} \mathrm{C}\right)$, um diagrama ternário é apresentado na Figura 2 sendo estudados $\mathrm{O}$ GLYCEROL, METHYL-O e METHANOL.

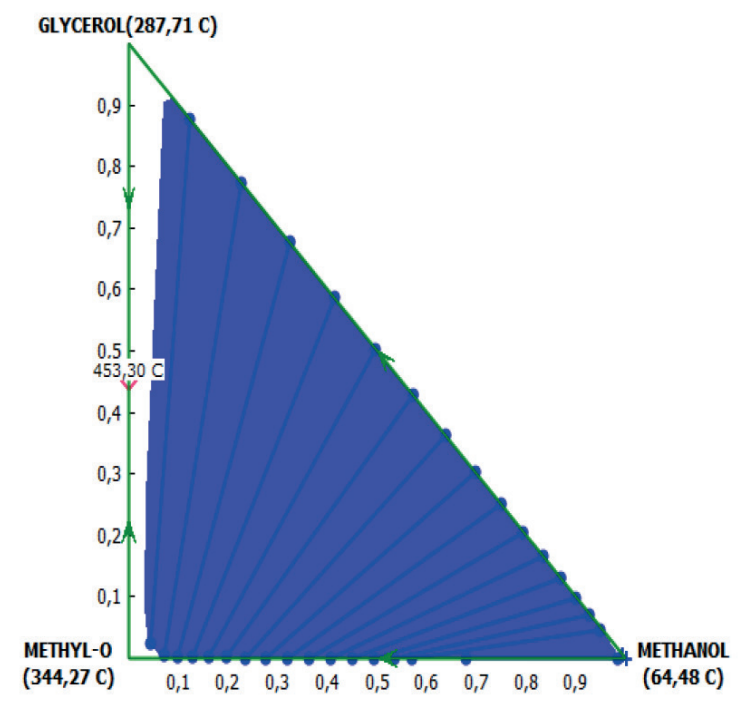

Figura 2. Diagrama GLYCEROL, METHYL-O e METHANOL.

O azeotropo apresenta uma base molar atingida de 0,4516 e 0,5484 para o GLYCEROL E METHYL-O respectivamente.

O estudo termoquímico desenvolvio apresenta o plano de trabalho e comportamento dos componentes para o planejamento e desenho de uma unidade de síntese do biodiesel, especificamente uma unidade reativa, atingindo a possibilidade de obtenção do biodiesel e achando limitações na separação pela presença do azeótropo. O GLYCEROL e METHYL-O descrevem um comportamento azeotrópico a 453 ${ }^{\circ} \mathrm{C}$ atingindo uma composição molar de 0,4516 e 0,5484 respectivamente.

\section{Conclusões}

As particularidades dos sistemas, o comportamento azeotrópico das misturas foram identificadas através do simulador de processos Aspen Plus V9 levando em conta o estudo dos fenómenos e das dificuldades da mistura de componentes na produção do TAME.

As particularidades do sistema e o comportamento azeotrópico foi identificado para o processo de obtenção de biodiesel. O método UNIFAC-DMD permitiu estimar o equilíbrio com precisão para o processo de BIODIESEL. As pressões de vapor foram fundamentais para o equilíbro de fases, sendo que as condições do processo estão em função dos pontos críticos dos componentes e da temperatura onde se apresentam os azeótropos. 
É identificado que o GLYCEROL e METHYL-O descrevem um comportamento azeotrópico a $453^{\circ} \mathrm{C}$ atingindo uma composição molar de 0,4516 e 0,5484 respectivamente.

\section{Agradecimentos}

Os autores agradecem o apoio da Universidad EAN, UNIFESP, UNICAMP, UTB e Universidad de Cartagena,

\section{Referências}

[1] Gebremariam SN, Marchetti JM. Economics of biodiesel production. Energy Conversion and Management. 2018;168:74-84.

[2] Meng K, Wu Y, Lin Q, Shan F, Fu W, Zhou K, et al. Microexplosion and ignition of biodiesel/ ethanol blends droplets in oxygenated hot co-flow. Journal of the Energy Institute. 2019;92(5):1527-36.

[3] Chen J, Li J, Dong W, Zhang X, Tyagi RD, Drogui $P$, Surampalli RY. The potential of microalgae in biodiesel production. Renewable and Sustainable Energy Reviews. 2018;90:336-46.

[4] Zhang Y, Huang R, Huang Y, Huang S, Zhou $P$, Chen $X$, Qin T. Experimental study on combustion characteristics of an n-butanolbiodiesel droplet. Energy. 2018;160:490-9.

[5] Ambat I, Srivastava V, Sillanpää M. Recent advancement in biodiesel production methodologies using various feedstock: A review. Renewable and Sustainable Energy Review S. 2018;90:356-69.

[6] Baldea M, Edgar TF. Dynamic process intensification. Current opinion in chemical engineering. 2018;22:48-53.

[7] Selaimia R, Beghiel A, Oumeddour R. The synthesis of biodiesel from vegetable oil. Procedia-Social and Behavioral Sciences. 2015;195:1633-8.

[8] Deng Y, Hu X, Cheng L, Wang H, Duan L, Qiu R. Zirconocene-catalysed biodiesel synthesis from vegetable oil with high free fatty acid contents. Journal of Organometallic Chemistry. 2018;870:116-20.

[9] Humphrey JL, SeibertAF, Koort RA. Separation technologies: Advances and priorities. United States of America: Humphrey (JL) and
Associates; 1991.

[10] Wright RO. Fractionation apparatus. US Patent 2.471.134.1949 May 24.

[11] Romagnoli ÉS, Borsato D, Silva LRC, Chendynski LT, Angilelli KG, Canesin EA. Kinetic parameters of the oxidation reaction of commercial biodiesel with natural antioxidant additives. Industrial Crops and Products. 2018; 125:59-64.

[12] Xuan J, Leung MKH, Leung YCD, Meng L. A review of biomass-derived fuel processors for fuel cell systems. Renewable and Sustainable Energy Reviews. 2008;13(7-8):1-13.

[13]Demirbas A. Progress and recent trends in biodiesel fuels. Energy Conversion and Management. 2009;50(1):14-34.

[14] Gmehling J, Jiding L, Schriller M. A modified UNIFAC model. 2. Present parameter matrix and results for different thermodynamic properties. I\&EC research. 1993;32(1):178-93.

[15] Gmehling J, Li J, Schiller M. A modified UNIFAC model. 2. Present parameter matrix and results for different thermodynamic properties. I\&EC research. 1993;32(1):178-93.

[16] Wittig R, Lohmann J, Gmehling J. Prediction of phase equilibria and excess properties for systems with sulfones. AIChE Journal. 2003;49(2):530-37.

[17] Jakob A, Grensemann H, Lohmann J, Gmehling J. Further Development of Modified UNIFAC (Dortmund): Revision and Extension 5. I\&EC research. 2006;45(23):7924-33.

[18] Narvaez PC, Rincon SM, Sanchez FJ. Kinetics of Palm Oil Methanolysis. Journal of American Oil Chemical Society. 2007;84:971-77.

[19] Fredenslud Aa, Gmehling J, Rasmussen P. Vapor-liquid Equilibria Using UNIFAC. A Group Contribution Model.The Netherlands: Elsevier science publishers B.V;1977.

[20] Pulido JL, Maciel MRW, Filho RM. Nuevas Perspectivas en Procesos de Separación: Simulación Columna de Destilación con Integración Interna de Calor (CDIIC). rev.ion. 2010;23(1):7-12.

[21] Pulido JL, Cespedes IDS, Delgado ADG, Navas JB, Concha VOC, Nunhez JR. Estudo Termoquímico na Produção de Aditivo de Gasolina Tert-Amyl-Methyl-Ether (TAME). rev. ion. 2019;32(1):87-95. 